\title{
Evidências de Validade do Bender - Sistema de Pontuação Gradual (B-SPG)
}

\author{
Evidences of Validity of Bender - Gradual Scoring System (B-SPG)
}

\author{
Ana Paula Porto Noronha*, Acácia Aparecida Angeli dos Santos \& Fermino Fernandes Sisto \\ Universidade São Francisco, Itatiba, Brasil
}

\begin{abstract}
Resumo
Esse estudo objetivou buscar evidências de validade para o teste de Bender - Sistema de Pontuação Gradual ( $B$ $S P G)$, por meio da investigação das mudanças desenvolvimentais, expressas pela relação entre os escores com a idade. Fizeram parte do estudo 1052 crianças de oito escolas públicas estaduais e municipais de Campinas e região, de pré-escola à $4^{\mathrm{a}}$ série do ensino fundamental. As idades variaram entre 6 e 10 anos, com uma média de 8,35 anos. Os achados revelaram que conforme aumentou a idade, diminuiu a pontuação obtida pelo $B-S P G$, indicando que com o aumento da idade, as distorções da cópia progressivamente diminuem. Embora outros estudos sejam recomendados, os resultados aqui apresentados estão em consonância com trabalhos que buscaram a constatação de que é possível encontrar um caráter maturacional nas figuras do teste.

Palavras-chave: Avaliação psicológica; testes psicológicos; parâmetros psicométricos.
\end{abstract}

\begin{abstract}
This study aimed to gather evidences of validity for the Bender test - Gradual Scoring System (B-SPG), investigating developmental changes based on the relationships between its scores and the ages of the children. The study investigated 1052 children attending from pre-school to fourth grade of eight municipal and state public schools of Campinas (a city in the state of São Paulo, Brazil) and the surrounding region. Ages ranged from six to ten years old, with an average of 8,35. The findings indicated that according to the increase of age, the $B-S P G$ scores diminished, suggesting that the distortions progressively diminish as the children become older. Although other studies are suggested, the results presented here are consonant with researches that sought to establish that it is possible too find a maturational character among the figures of the test.

Keywords: Psychological assessment; psychological tests; psychometrics parameters.
\end{abstract}

O teste de Bender consta dentre os instrumentos mais utilizados na avaliação psicológica, como pode ser verificado em revisões realizadas nos países estrangeiros (Buckley, 1978; Lubin \& Sands, 1992; Sangster, Rogers \& Searight, 1993). Também no plano nacional há indicativos de que o teste é bastante conhecido e utilizado tanto por profissionais como por estudantes de psicologia (Noronha, 2002; Noronha et al., 2002).

Estudiosos de várias nacionalidades têm apontado o Bender como um instrumento válido para muitos usos e contextos. Dentre eles, destaca-se a avaliação do desenvolvimento percepto-motor (Harper, 1998; Imm, Foster, Belter \& Finch Jr., 1991; McCarron \& Horn, 1979; McIntosh, Belter, Saylor, Finch Jr. \& Edwards, 1988; Sohlberg, 1985); do diagnóstico diferencial de crianças com problemas de aprendizagem (Arrillaga, Eschebarria \& Goya, 1981; Goldstein, Petterson \& Sheaffer, 1989; Jing, Deqing \& Longhui, 2001; Mallinger \& Longley, 1988); para predizer habilidades de leitura e matemática (Cunha, 2000; Lesiak,

* Endereço para correspondência: Rua Alexandre Rodrigues Barbosa, 45, Centro, Itatiba, SP, 13.253-231.E-mail:ana.noronha@saofrancisco.edu.br
1984; Nielson \& Sapp, 1991); e para avaliar memória e desenvolvimento (Finch Jr., Spirito, Garrison \& Marshall, 1983; Hutton, 1966).

No Brasil, também se encontra o amplo espectro de temas investigados. Dentre eles, várias pesquisas utilizaram o Bender em grupos com dificuldades específicas, quais sejam, estudos com pacientes epilépticos e esquizofrênicos (Maciel Jr. \& La Puente, 1983); em crianças com deficiência auditiva (Cariola, Piva, Yamada \& Bevilacqua, 2000; Gemignani, \& Chiari, 2000) ou em pacientes portadores da síndrome de imunodeficiência adquirida (Mattos, 1991); em relação a traços de personalidade (Sisto, Bueno \& Rueda, 2003); dificuldades de aprendizagem (Suehiro \& Santos, 2005) e rendimento escolar (Bandeira \& Hutz, 1994). Há ainda, os estudos de validação de $\operatorname{Kroeff}(1988,1992)$, utilizando-se do método de correção proposto por Koppitz. Nesse particular, o autor apresentou normas específicas para escolas públicas e privadas brasileiras e analisou a diferença de resultados de acordo com a série.

Ao refletir sobre a obra de Bender, Koppitz (1989) ressalta que para ela a função gestáltica está associada a diversas outras funções cognitivas, tais como percepção visual, habilidade motora manual, conceitos temporais e espaciais 
e organização ou representação. Ademais, a percepção e a reprodução das figuras gestálticas é influenciada por princípios biológicos e de ação sensório-motora, que variam entre indivíduos, em razão do padrão de desenvolvimento de cada um, bem como de eventuais alterações patológicas funcionais ou orgânicas. A medida da maturidade percepto-motora, tal como medida pelo Bender, também foi relacionada com a capacidade de linguagem e memória por Schachter, Brannigan e Tooke (1991).

Especialmente no que diz respeito ao uso de padrões com diferentes graus de complexidade e princípios de organização para a mensuração da maturidade perceptomotora, Bender (1974) justifica que a Figura $A$ foi determinada como a primeira em razão da simplicidade da reprodução de duas partes (losango e círculo), o que permitiria ao sujeito, identificar com facilidade suas gestalts. Ainda nesse sentido, a Figura 1 seria percebida como uma série de pares de pontos, ao mesmo tempo em que a Figura 2 seria identificada como uma série de linhas oblíquas compostas de três unidades, inclinadas desde a esquerda acima até a direita abaixo.

A gestalt das proximidades está determinada também na Figura 3, de tal modo que o desenho se assemelha a uma ponta de flecha. Já a Figura 4 se estrutura pelo princípio da continuidade da organização geométrica, por meio da representação de um quadrado aberto na parte superior e de uma onda interceptando um dos vértices dele. Em alguma medida, um princípio similar rege as Figuras $A$ e 5 , diferentemente da Figura 6, formada por linhas sinuosas de diferentes tamanhos de onda que se entrecortam obliquamente. Por fim, as Figuras 7 e 8 são duas configurações compostas pelas mesmas unidades, embora freqüentemente não sejam observadas como tal, pois na última prevalece o princípio da continuidade das formas geométricas, enquanto na primeira elas se tangenciam.

No que se refere ao desenvolvimento, Paim (1992) sustenta que aos 6 anos a percepção visual permite a elaboração de laçadas e linhas, de forma que a criança já é capaz de reproduzir corretamente as figuras A, 1, 4 e 5. Aos sete anos, a criança faz as linhas oblíquas nas figuras A e 8, assim como une as subpartes mais satisfatoriamente. O refinamento dos pontos e os contornos mais uniformes acontecem aos oito anos, enquanto aos dez anos, os hexágonos são realizados com êxito.

Com vistas a avaliar a ampla faixa etária avaliada pelo Bender, incluindo crianças mais novas, Mazzeschi e Lis (1999) aplicaram o instrumento em 1065 crianças com idade variando entre 3 anos e 5 meses a 11 anos e 4 meses. As autoras focalizaram os erros apresentados em cada desenho e sua relação com a pontuação geral, fruto da soma dos erros em todas as figuras, de acordo com os critérios preconizados por Koppitz (1975). Os achados revelaram que à medida que a idade aumentou, a média de erros diminuiu, o que está em consonância com os trabalhos de Arrillaga, Eschebarria e Goya (1981), Jing, Deqing e Longhui (2001) e $\operatorname{Kroeff}(1988,1992)$; em comum, apontaram o teste como sensível para diferenciação das idades.
No que respeita ao aspecto maturacional do teste, destaca-se o trabalho realizado por Moura (1982) com 160 sujeitos de 3 a 6 anos. Foram avaliadas as diferenças da performance dos grupos etários, tendo ficado evidente o caráter evolutivo na reprodução de figuras geométricas. No entanto, não foram essas as idades inicialmente estudadas por Koppitz.

As pesquisas de Brannigan e Brunner $(1991,2002)$ e Moose e Brannigan (1997) consideraram o sistema falho para a captação de diferenças entre crianças da faixa etária para a qual o teste foi destinado. Em conseqüência, muitos autores têm trabalhado numa versão modificada do teste, bem como na proposta de um outro sistema de avaliação que considerasse aspectos mais qualitativos do desenho produzido pelos testandos.

Com base em estudo realizado com 652 crianças norteamericanas Taylor, Kauffman e Partenio (1984) questionaram a validade do sistema na identificação de características desenvolvimentais em crianças com mais de oito anos. O estudo de adolescentes por meio do sistema Koppitz também foi referido (Pascal \& Suttell, 1951, entre outros). No entanto, houve evidências que demonstraram que ele é inapropriado para aplicação em crianças acima de 11 anos, visto que elas apresentavam um patamar, a partir do qual não foi possível uma discriminação das idades (Shapiro \& Partenio, 1995).

No Brasil, vários trabalhos foram desenvolvidos apontando as fragilidades do sistema Koppitz (1975). O estudo realizado com crianças do estado no Rio de Janeiro por Britto e Santos (1996) gerou resultados que endossaram a recomendação de cautela na utilização das normas propostas para a discriminação de variáveis relacionadas à idade e ao sexo aqui no Brasil.

Nessa mesma linha, está a pesquisa desenvolvida por Sisto, Noronha e Santos (2004) com crianças das séries iniciais do ensino fundamental. Os autores observaram a existência de correlação negativa entre idade e deformação em algumas figuras, o que era esperado, pois conforme aumentou a idade, diminuiu a intensidade da deformação. Nada obstante, os resultados não foram os esperados a ponto de caracterizar o sistema como uma medida sensível ao caráter maturacional do desenvolvimento infantil. Em acréscimo, os valores de alfa indicaram consistência interna frágil.

Em outro estudo, Sisto, Santos e Noronha (2004) analisaram os critérios de Koppitz para avaliar a integração das figuras do Bender e seu caráter evolutivo. Os participantes foram escolares de ambos os sexos, de 7 a 10 anos. Os resultados indicaram baixa correlação entre idade e número de erros. Entretanto, apenas dois grupos etários foram diferenciados significativamente pelo teste ad-hoc de Tukey mesmo quando as análises de variância apontaram diferenças significativas. A análise das correlações item-total mostrou problemas de ajustamento, já que apenas duas medidas apresentaram relação significativa com a pontuação total.

Apesar de muito utilizado e de ter gerado muitas pesquisas que vão ao encontro dos resultados apontados por Koppitz (1975), o sistema da autora também sofreu críticas 
contundentes. Buckley (1978) concluiu que faltam evidências de validade para muitos dos usos para os quais é indicado, como por exemplo, para a predição de rendimento escolar ou problemas neurológicos. O valor dos critérios de correção utilizados foi questionado por autores como Shapiro e Partenio (1995), Taylor, Kauffman e Partenio (1984), por exemplo, com a afirmação de que há limitações na interpretação da pontuação obtida, com conseqüentes problemas para sua utilização no diagnóstico neuropsicológico, bem como na identificação de aspectos desenvolvimentais.

De forma geral o caráter evolutivo do Teste do Bender tem sido observado na maioria das pesquisas pesquisadas. Da literatura sobre o Bender depreende-se, como já referido, que é um instrumento bastante usado no mundo e no Brasil. No entanto, há muitos estudos que apresentam críticas em relação a suas evidências de validade, com controvérsias entre os autores (Bandeira \& Hutz, 1994; Kroeff, 1988, 1992; Moura, 1982; Pinelli Jr., 1990; Pinelli Jr. \& Pasquali, 1990; Sisto, Noronha \& Santos, 2004; Sisto, Santos \& Noronha, 2004).

No Brasil, o sistema desenvolvido por Koppitz era freqüentemente empregado até a promulgação das Resoluções do Conselho Federal de Psicologia (CFP, 2001, 2003), que estabeleceu princípios sobre a elaboração, o uso e a comercialização de testes psicológicos. A partir de então, o Bender não pôde mais ser usado para fins diagnósticos, já que não possuía estudos de validade, precisão e normatização com amostras brasileiras, o que reafirmou a necessidade de novas pesquisas. Possivelmente isso justifique o desenvolvimento do teste Gestáltico Visomotor de Bender - Sistema de Pontuação Gradual, publicado recentemente (B-SPG) por Sisto, Noronha e Santos (2006), tendo como embasamento os pressupostos teóricos de Bender (1955).

O B-SPG objetiva a avaliação da distorção de forma, compreendida como o desrespeito aos aspectos estruturais do desenho, de modo tal que pontos, linhas, retas, curvas e ângulos são desenhados sem precisão (Paim, 1992; Sisto, Noronha \& Santos, 2006). O sistema utiliza as mesmas nove figuras eleitas por Bender, como as mais representativas para avaliar a maturidade viso-motora, especialmente o que se refere à função pela qual o indivíduo responde gestalticamente a uma série de estímulos.

Tendo em vista o exposto, este estudo destinou-se a buscar evidências de validade para o teste de Bender - Sistema de Pontuação Gradual $(B-S P G)$. Nesse sentido, propôs-se a investigar mudanças desenvolvimentais expressas pela diminuição nos escores de acordo com o aumento da idade.

\section{Método}

\section{Participantes}

Fizeram parte do estudo 1052 crianças oriundas de oito escolas públicas estaduais e municipais de Campinas e região. Os participantes foram 116 crianças da pré-escola (11,02\%), 177 da $1^{\text {a }}$ série $(16,83 \%), 319$ da $2^{\text {a }}(30,32 \%)$, 189 da $3^{\text {a }}(17,97 \%)$ e 251 da $4^{\text {a }}$ série $(23,86 \%)$ do ensino fundamental. As idades variaram entre 6 e 10 anos, com uma média de 8,35 anos (moda e mediana de 8 anos) e um desvio padrão de 1,29. Em relação ao sexo, 51,1\% eram meninas.

\section{Material}

Folha de sulfite em branco e os modelos das nove figuras de Bender em transparência (para aplicação coletiva) e cartões com as mesmas figuras para aplicação individual. Pelo Sistema de Pontuação Gradual (Bender-SPG) atribui-se uma nota de zero até três pontos, de acordo com a qualidade do desenho, de tal modo que nos desenhos mais pontuados, o erro é mais grave ou acentuado conforme descrição e ilustração do manual (Sisto, Noronha \& Santos, 2006).

\section{Procedimento}

Depois de aprovado pelo comitê de ética e após o consentimento dos pais, as crianças foram submetidas à aplicação do instrumento. Em pelo menos uma classe de cada série as figuras do Bender foram aplicadas individualmente por alunos de graduação e pós-graduação em psicologia, especialmente treinados para a tarefa. Para os demais participantes a aplicação foi coletiva por meio de transparências apresentadas em um retroprojetor.

\section{Resultados}

A investigação do caráter evolutivo foi um dos interesses na proposta para o Bender - Sistema de Pontuação Gradual $(B-S P G)$. Para tanto, dois aspectos foram observados, quais sejam, primeiramente as correlações entre idade e a pontuação do $B-S P G$ e depois, as diferenças de médias dos grupos e a possibilidade de discriminação entre eles. Vale destacar que foram utilizadas duas medidas distintas para a análise, uma que se refere à média da pontuação bruta, ou seja, a soma dos pontos atribuídos, e, outra, a medida Rasch.

No que respeita à correlação entre idade e pontuação e medida Rasch no $B-S P G$ os índices obtidos foram de $r=-$ 0,58 e $r=-0,60$, respectivamente, ambos significativos a $p<0,001$. Assim, os achados revelam que conforme aumentou a idade, diminuiu a pontuação ou a medida Rasch, obtidas pelos $B-S P G$. Em outros termos, com o aumento da idade as distorções da cópia vão progressivamente diminuindo, e há uma conseqüente melhora das reproduções ao longo das idades. A Figura 1, na próxima página, permite a ilustração dessa tendência.

No que tange ao estudo sobre as diferenças de médias dos grupos e sua possibilidade de discriminação, foram usadas a análise de variância e a prova ad-hoc de Tukey. No caso da análise de variância, levou-se em consideração tanto a idade quanto o sexo (4X2). Os resultados encontram-se na Tabela 1 , na próxima página.

Para ambas as avaliações, a idade foi a fonte de maior variância $\quad[F(4,1048)=139,07 ; \quad p<0,001] \quad$ e $[F(4,1048)=155,15 ; p<0,001]$ para as medidas Rasch $\mathrm{e}$ pontuações brutas, respectivamente, além de estatisticamente significativa. Sexo não se mostrou como fonte de variância 


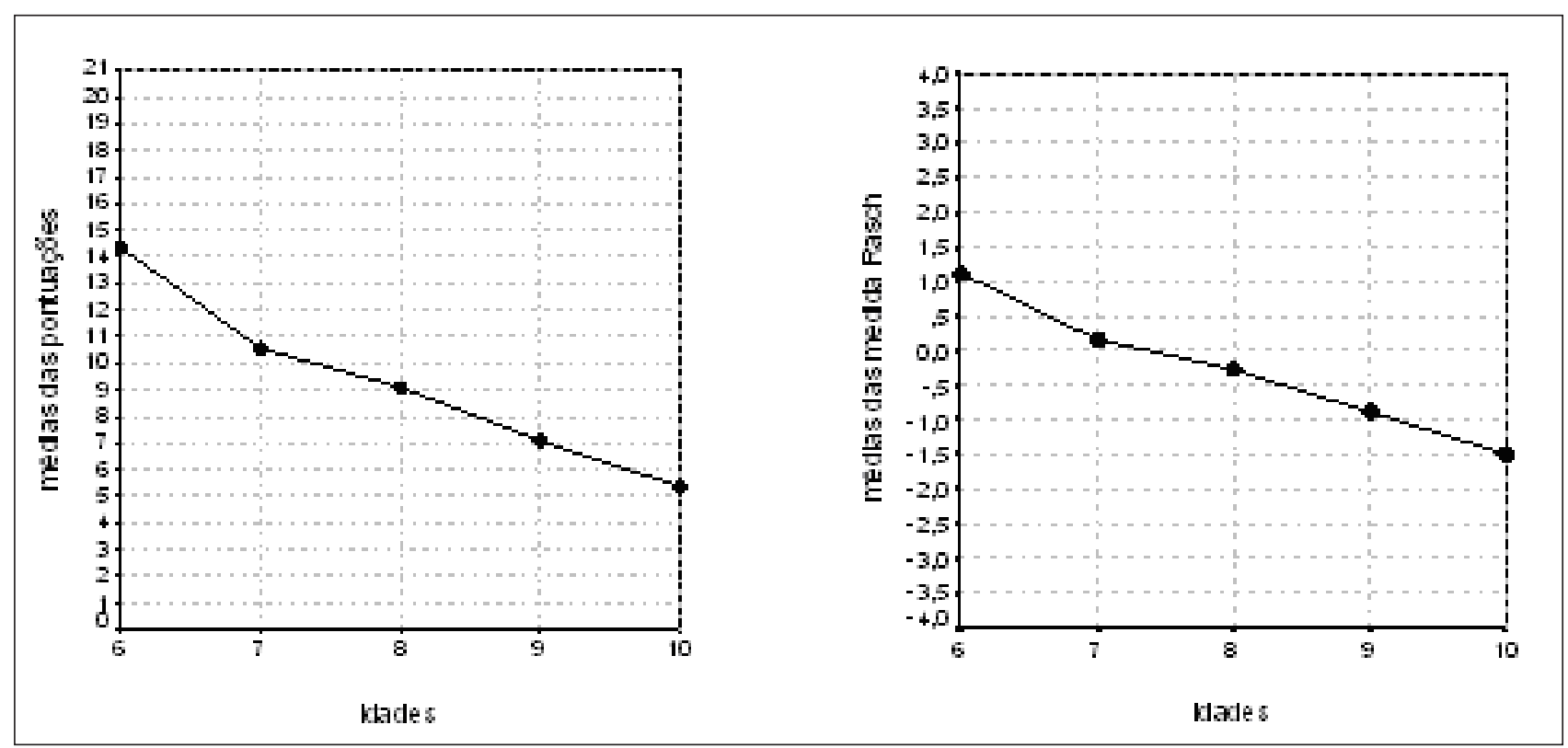

Figura 1. Médias nas avaliações em relação às idades.

Tabela 1

Valores de F, p e Graus de Liberdade (gl) em Relação à Idade, Sexo e Efeito de Interação com Base na Medida Rasch e na Pontuação

\begin{tabular}{lcrcrrc}
\hline & \multicolumn{3}{c}{ Medida Rasch } & \multicolumn{3}{c}{ Pontuação } \\
\hline Fonte & $g l$ & $F$ & $p$ & $g l$ & $F$ & $p$ \\
\hline Idade & 4 & 139,07 & 0,000 & 4 & 155,15 & 0,000 \\
Sexo & 2 & 0,56 & 0,570 & 2 & 0,69 & 0,504 \\
Idade * Sexo & 4 & 2,58 & 0,036 & 4 & 2,33 & 0,054 \\
\hline
\end{tabular}

suficiente para produzir diferença que não fosse aleatória. Por fim, a interação entre idade e sexo mostrou-se significativa em relação à medida Rasch, mas não em relação à pontuação. Para uma melhor visualização desse resultado é apresentada a Figura 2.

No que se refere à diferença entre sexos, também observada na Figura 2, para ambas as medidas (médias brutas e Rasch), em dois pontos há certa assimetria entre os resultados dos meninos e das meninas. Uma se dá aos sete anos,

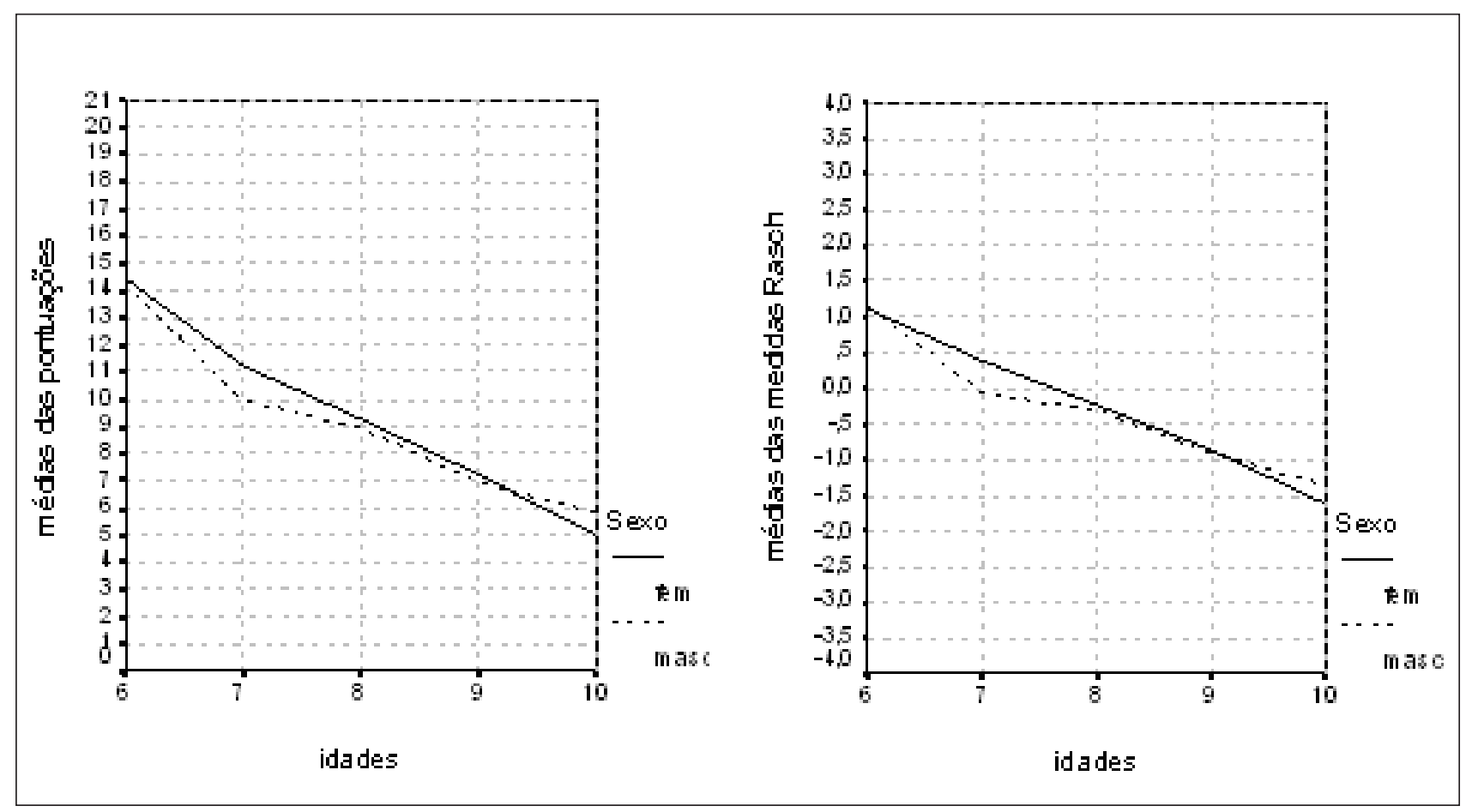

Figura 2. Médias da pontuação bruta e da medida Rasch por idade e sexo. 
quando ocorre uma diferença maior entre eles e, a outra, aos dez anos quando por primeira vez na cronologia estudada os meninos obtêm uma média de erros maior que as meninas. A fim de uma maior investigação, calculou-se valores de $t$ independentemente de idade e levando-as em consideração. Esses resultados podem ser encontrados na Tabela 2.

Tabela 2

Médias, Valores de te p, para Sexo, em Relação às Idades e o Tipo de Escore (pontuação e medida Rasch)

\begin{tabular}{|c|c|c|c|c|c|}
\hline Idades & Tipos de medida & Sexo & Médias & $t$ & $p$ \\
\hline \multirow{4}{*}{ Todas } & \multirow{2}{*}{ Medida Rasch } & $M$ & $-0,49$ & \multirow{2}{*}{$-0,01$} & \multirow{2}{*}{0,996} \\
\hline & & $F$ & $-0,49$ & & \\
\hline & \multirow{2}{*}{ Pontuação } & M & 8,51 & \multirow{2}{*}{$-0,23$} & \multirow{2}{*}{0,818} \\
\hline & & $F$ & 8,57 & & \\
\hline \multirow{4}{*}{6 anos } & \multirow{2}{*}{ Medida Rasch } & $M$ & 1,14 & \multirow{2}{*}{0,12} & \multirow{2}{*}{0,906} \\
\hline & & $\mathrm{F}$ & 1,12 & & \\
\hline & \multirow{2}{*}{ Pontuação } & M & 14,26 & \multirow{2}{*}{$-0,30$} & \multirow{2}{*}{0,768} \\
\hline & & $\mathrm{F}$ & 14,46 & & \\
\hline \multirow{4}{*}{7 anos } & Men & $M$ & $-0,06$ & \multirow{2}{*}{$-2,46$} & \multirow{2}{*}{0,015} \\
\hline & Miea & $\mathrm{F}$ & 0,38 & & \\
\hline & \multirow{2}{*}{ Pontuação } & M & 9,97 & \multirow{2}{*}{$-2,17$} & \multirow{2}{*}{ 0,031 } \\
\hline & & $\mathrm{F}$ & 11,25 & & \\
\hline \multirow{4}{*}{8 anos } & Med & $M$ & $-0,31$ & \multirow{2}{*}{$-0,76$} & \multirow{2}{*}{0,449} \\
\hline & Nieat & $\mathrm{F}$ & $-0,22$ & & \\
\hline & \multirow{2}{*}{ Pontuação } & M & 8,94 & \multirow{2}{*}{$-0,75$} & \multirow{2}{*}{0,454} \\
\hline & & $\mathrm{F}$ & 9,25 & & \\
\hline \multirow{4}{*}{9 anos } & \multirow{2}{*}{ Medida Rasch } & $M$ & $-0,91$ & \multirow{2}{*}{$-0,15$} & \multirow{2}{*}{0,882} \\
\hline & & $\mathrm{F}$ & $-0,88$ & & \\
\hline & \multirow{2}{*}{ Pontuação } & M & 6,95 & \multirow{2}{*}{$-0,58$} & \multirow{2}{*}{0,566} \\
\hline & & $\mathrm{F}$ & 7,25 & & \\
\hline \multirow{4}{*}{10 anos } & Medida Rasch & $M$ & $-1,37$ & 180 & 0073 \\
\hline & 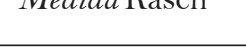 & $\mathrm{F}$ & $-1,63$ & $1, \mathrm{OU}$ & 0,070 \\
\hline & Pont & M & 5,79 & 2,11 & 5 \\
\hline & & $\mathrm{F}$ & 4,99 & 2,11 & \\
\hline
\end{tabular}

Embora tenham sido identificadas diferenças significativas aos sete anos, para ambas as medidas e aos dez, apenas na medida Rasch, no geral, em nenhuma análise a variável sexo mostrou produzir diferenças significativas nos dados. Assim, a análise pela prova de Tukey foi feita independentemente de idades. Os resultados podem ser examinados na Tabela 3, e assim como nas análises anteriores, indicam a pontuação bruta e a medida Rasch. Em ambas as medidas, houve a formação de cinco subconjuntos, um para cada faixa de idade.

\section{Discussão}

O caráter de maturidade visomotora do estudo original de Bender (1955) foi observado nos diferentes estudos apresentados para se construir sistema para avaliação das figuras inicialmente selecionadas por ela. Essa característica parece ser de grande valia para o teste, principalmente para os profissionais interessados em analisar aspectos psiconeurológicos associados a esse tipo de maturidade. Nesse ensejo, a autora acreditava que a maioria das crianças aos 11 anos conseguiria realizar a cópia das figuras sem erro e que o aspecto maturacional estava contemplado nos diferentes estímulos representados pelas nove figuras.

As análises aqui apresentadas permitem conjecturas no sentido de que a idade está correlacionada com a maturidade percepto-motora, entendida mais especialmente nesse estudo, como o desrespeito aos aspectos estruturais do desenho, conforme já afirmado anteriormente (Paim, 1992; Sisto, Noronha \& Santos, 2006). Ainda nessa perspectiva, as análises evidenciaram que os grupos de idade se diferenciaram, de forma significativa nas medidas brutas e na medida Rasch.

Os achados do estudo com o B-SPG estão em consonância com outros trabalhos que buscaram a constatação de que é possível encontrar um caráter maturacional nas figuras do teste (Harper, 1998; Imm, Foster, Belter \& Finch Jr., 1991; McCarron \& Horn, 1979; McIntosh, Belter, Saylor, Finch Jr. \& Edwards, 1988; Sohlberg, 1985). No

Tabela 3

Subconjuntos formados pela Prova de Tukey em Razão das Idades para a Medida Rasch (MR) e Pontuações (Pont) no B-SPG

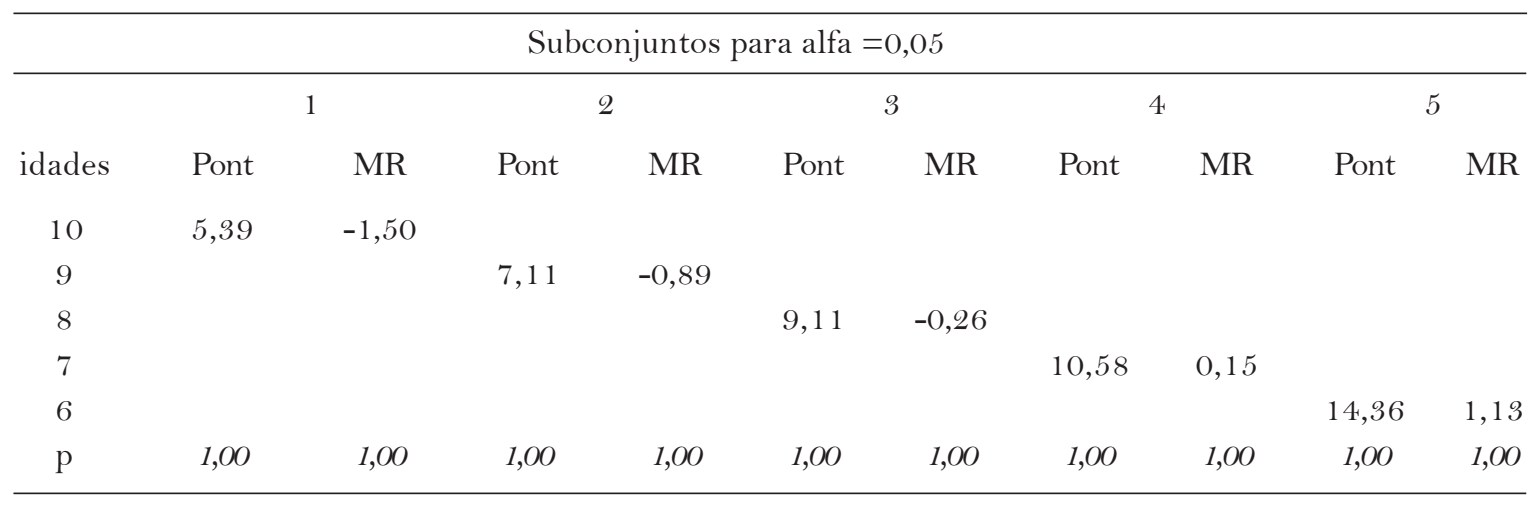


entanto, deve-se ter em conta que para que isso possa ser observado, faz-se necessário um sistema de correção que permita essa análise apurada. As pesquisas destacadas na fundamentação desse estudo evidenciaram críticas quanto aos respectivos resultados, quando do uso do Sistema de Koppitz (Brannigan \& Brunner, 1991, 2002; Moose \& Brannigan, 1997; Pascal \& Suttell, 1951), o que reforça a necessidade da continuidade de pesquisas que abordem a construção de critérios de pontuação.

Quanto à comparação das pontuações entre os sexos, os resultados sugeriram que não houve fortes evidências de que meninos e meninas se diferenciam na maturidade percepto-motora. Nesse sentido, valem as asserções de Britto e Santos (1996), que endossaram a recomendação de cautela na utilização das normas propostas para a discriminação de variáveis relacionadas à idade e ao gênero, sem que haja estudos as que fundamentem.

\section{Conclusão}

Tendo em vista os objetivos do estudo, cabe destacar que foram atendidos, visto que se observou a sensibilidade do $B-S P G$ para a captação do caráter maturacional do desenvolvimento psicomotor. Outras análises poderiam ser feitas, no tocante aos padrões de dificuldade das figuras do teste e a relação deles com as faixas etárias, o que seria relevante para o desenvolvimento de estudos futuros com o instrumento, mais especialmente com o sistema de correção $(B-S P G)$.

Ainda, a título de finalização, esse estudo pretendeu colaborar com o rol de pesquisas sobre parâmetros psicométricos dos testes psicológicos. Acredita-se que trabalhos dessa natureza devam ser realizados com mais freqüência pela comunidade científica, a fim de que se promova o uso mais adequado e a avaliação psicológica de excelência.

\section{Referências}

Arrillaga, S. G. P., Eschebarria, C. C., \& Goya, I. O. (1981). Una investigación sobre aspectos intelectuales en una población de deficientes de un grupo marginal. Psiquis: Revista de Psiquiatria, Psicologia y Psicosomatica, 2(1), 12-22.

Bandeira, R. D., \& Hutz, C. S. (1994). A contribuição dos testes $\mathrm{DFH}$, Bender e Raven na predição do rendimento escolar na primeira série. Psicologia: Teoria e Pesquisa, 10(1), 59-72.

Bender, L. (1955). Test Guestáltico Visomotor(D. Carnelli, Trad.). Buenos Aires, Argentina: Paidós.

Bender, L. (1974). Test Guestáltico Visomotor. Buenos Aires, Argentina: Paidós.

Brannigan, G. G., \& Brunner, N. A. (1991). Relationship between two scoring systems for the modified version of the Bender Gestalt test. Perceptual and Motor Skills, 72, 286.

Brannigan, G. G., \& Brunner, N. A. (2002). Guide to the qualitative scoring system for the modified version of the Bender-Gestalt Test. Springfield, IL: Charles C. Thomas.

Britto, G. N. O., \& Santos, T. R. (1996) The Bender Gestalt test for 5-to 15-year old Brazilian children: Norms and validity. Jornal de Medicina e Biologia, 29(11), 1513-1518.
Buckley, P. D. (1978). The Bender Gestalt Test: A review of reported research with school-age subjects, 1966-1977. Psychology in the Schools, 15(3), 327-335.

Cariola, T. C., Piva, R. A., Yamada, M. O., \& Bevilacqua, M. C. (2000). A prova gráfica de organização perceptiva para crianças de quatro a seis anos deficientes auditivas. Pediatria Moderna, 36(9), 588-594.

Conselho Federal de Psicologia. (2001). Resolução no 025/2001. Retirado em 21 nov. 2001, de http://www.pol.org.br

Conselho Federal de Psicologia. (2003). Resolução $n^{\circ}$ 02/2003. Retirado em 06 dez. 2003, de http://www.pol.org.br

Cunha, J. A. (2000). Psicodiagnóstico - V. Porto Alegre, RS: Artes Médicas.

Finch, A. J., Jr., Spirito, A., Garrison, S., \& Marshall, P. (1983). Developmental differences in Bender-Gestalt recall of children with learning and behavior problems. Perceptual and Motor Skills, 56, 87-90.

Gemignani, E. Y. M. C., \& Chiari, B. M. (2000). Escala de maturação do Teste de Bender em um grupo de crianças deficientes auditivas. Pró-fono, 12(2), 49-53.

Goldstein, D. J., Petterson, N. C., \& Sheaffer, C. I. (1989). Concurrent validity of the Gardner Test of visual-motor skills. Perceptual and Motos Skills, 69, 605-606.

Harper, H. K. (1998). Visual evoked potential correlates with visual-motor integration as measured by the Bender Gestalt Test. Dissertation Abstracts International, 58(8-B), 4509.

Hutton, J. B. (1966). Bender recall of children as related to age end intelligence. Perceptual and Motor Skills, 23, 34.

Imm, P. S., Foster, K. Y., Belter, R. W., \& Finch, A. J., Jr. (1991). Assessment of short-term visual memory in child and adolescent pschiatrics inpatients. Journal of Clinical Pschology, 47(3), 440443.

Jing, G., Deqing, T., \& Longhui, L. (2001). Visual-motor deficit in children with learning disabilities. Chinese-Mental-HealthJournal, 15(6), 388-390.

Koppitz, E. (1989). O Teste Gestáltico Bender para crianças. Porto Alegre, RS: Artes Médicas.

Koppitz, E. M. (1975). The Bender-Gestalt Test for young children: Research and application 1963-1973. New York: Grune \& Stratton.

Kroeff, P. (1988). Normas brasileiras para o Teste de Bender. Psicologia: Reflexão e Crítica, 1/2(3), 12-19.

Kroeff, P. (1992). Desempenho de crianças no Teste de Bender e nível sócio-econômico-cultutal. Psicologia: Reflexão e Crítica, 5(2), 119-126.

Lesiack, J. (1984). The Bender Visual Motor Gestalt test: Implications for the diagnosis and prediction of reading achievement. Journal of School Psychology, 22, 391-405.

Lubin, B., \& Sands, E. W. (1992). Bibliography of the psychometric properties of the Bender Visual-Motor Gestalt Test: 19701991. Perceptual and Motor Skills, 75, 385-386.

Maciel, J. A., Jr., \& La Puente, M. (1983). Avaliação multimodal do teste de Bender no psicodiagnóstico da epilepsia. Revista Brasileira de Neurologia, 2(19), 55-58.

Mallinger, B. L., \& Longley, K. F. (1988). Bip-Bender protocols of learning disabled and regular education students. Perceptual and Motor Skills, 67, 193-194.

Mattos, P. (1991). Os distúrbios mentais orgânicos e a síndrome de imunodeficiência adquirida. Jornal Brasileiro de Psiquiatria, $40(7), 375-381$. 
Mazzeschi, C., \& Lis, A. (1999). The Bender-Gestalt test: Koppitz’s Developmental Scoring System administered to two samples of Italian preschool and primary school children. Perceptual and Motor Skills, 88, 1235-1244.

McCarron, L. M., \& Horn, P. W. (1979). Haptic visual discrimination and intelligence. Journal of Clinical Psychology, 35(1), 117-120.

McIntosh, J. A., Belter, R. W., Saylor, C. F., Finch, A. J., Jr., \& Edwards, G. L. (1988). The Bender-Gestalt with adolescents: Comparision of two scoring systems. Journal of Clinical Psychology, 44(2), 226-230.

Moose, D., \& Brannigan, G. G. (1997). Comparison of preschool children's scores on the modified version of the Bender-Gestalt Test and the Developmental Test of Visual-Motor Integration. Perceptual and Motor Skills, 85(2), 766 - 775.

Moura, M. L. S. (1982). Reconhecimento e reprodução das figuras do Teste Gestaltico Visomotor-Bender: Um estudo evolutivo. Arquivos Brasileiros de Psicologia, 34(1), 50-61.

Nielson, S., \& Sapp, G. L. (1991). Bender-Gestalt developmental scores: Predicting reading and mathematics achievement. Psychologycal Reports, 69, 39-42.

Noronha, A. P. P. (2002). Problemas mais graves e mais freqüentes no uso dos testes psicológicos. Psicologia: Reflexão e Crítica, 15(1), 135-142.

Noronha, A. P. P., Oliveira, A. F., Cobêro, C., Paula, L. M., Cantalice, L. M., Guerra, P. B. C., Martins, R. M. M., \& Filizatti, R. (2002). Instrumentos psicológicos mais conhecidos por estudantes do sul de Minas Gerais. Avaliação Psicológica, 1(2), 151-158.

Paim, S. (1992). Diagnóstico e tratamento de problemas de aprendizagem. Porto Alegre, RS: Artes Médicas.

Pascal, G. R., \& Suttell, B. J. (1951). The Bender-Gestalt test, quantification and validity for adults. New York: Grune \& Stratton.

Pinelli, B., Jr. (1990). Adaptação do "Teste do Desenvolvimento da Integração Visomotora (VMI)" para uso no Brasil. Dissertação de Mestrado não-publicada, Universidade de Brasília, DF.

Pinelli, B., Jr., \& Pasquali, L. (1990). Parâmetros psicométricos do Teste Gestaltico Visomotor de Bender: Um estudo empírico. Revista de Psicologia, 1/2(7/8), 51-74.
Sangster, G., Rogers, B. J., \& Searight, H. R. (1993). The validity of Hutt's Bender-Gestalt scoring system for hostility: Correlation with the Interpessoal Behavior Survey's Agression Scales. Journal of Human Behavior, 30(2), 22-29.

Schachter, S., Brannigan, G. G., \& Tooke, W. (1991). Comparison of two scoring systems for the modified version of the BenderGestalt-Test. Journal of School Psychology, 29, 265-269.

Shapiro, S. K., \& Partenio, R. G. (1995). Koppitz scoring system as a measure of Bender-Gestalt performance in behaviorally and emotionally disturbed adolescents. Journal of Clinical Psychology, 51(1), 108-112.

Sisto, F. F., Bueno, J. M. H., \& Rueda, F. J. M. (2003). Traços de personalidade na infância e distorção e integração de formas: Um estudo de validade. Psicologia em Estudo, 8(1), 77-84.

Sisto, F. F., Noronha, A. P. P., \& Santos, A. A. A. (2004). Distorção de forma no Teste de Bender: Questionando seu critério de validade. Revista do Departamento de Psicologia da UFF, 16(2), 139-154

Sisto, F. F., Noronha, A. P. P., \& Santos, A. A. A. (2006). Teste Gestaltico Visomotor de Bender - Sistema de Pontuação Gradual $(B-S P G)$. São Paulo, SP: Vetor.

Sisto, F. F., Santos, A. A. A., \& Noronha, A. P. P. (2004). Critério de integração do Teste de Bender: Explorando evidências de validade. Avaliação Psicológica, 3(1), 13-20.

Sohlberg, S. C. (1985). Personality and neuropsychological performance of high-risk children. Schizophrenia-Bulletin, $11(1), 48-60$.

Suehiro, A. C. B., \& Santos, A. A. A. (2005) O Bender e as dificuldades de aprendizagem: Estudo de validade de critério. Avaliação Psicológica, 3, 2.

Taylor, R. L., Kauffman, D., \& Partenio, I. (1984). The Koppitz Developmental Scoring System for the Bender-Gestalt: Is it developmental? Psychology in the Schools, 21(4), 425-428.

Tosi, S. M. V. D. (1990). Adaptação Hutt do teste Gestaltico de Bender em alcoólicos e toxicômanos. Temas, 38, 108-127.

Recebido: 16/08/2006 Aceite final: 13/12/2006 